\title{
A Simplified Inverse Dynamics Modelling Method for a Novel Rehabilitation Exoskeleton with Parallel Joints and Its Application to Trajectory Tracking
}

\author{
Qianqian Fang $\mathbb{D}$, Ge Li $\mathbb{D}^{D}$, Tian Xu, Jie Zhao $\mathbb{D}$, Hegao Cai, and Yanhe Zhu $(\mathbb{D}$ \\ State Key Laboratory of Robotics and System, School of Mechatronics Engineering, Harbin Institute of Technology, Harbin, \\ Heilongjiang 150080, China \\ Correspondence should be addressed to Yanhe Zhu; yhzhu@hit.edu.cn
}

Received 3 September 2019; Revised 24 October 2019; Accepted 12 November 2019; Published 6 December 2019

Academic Editor: Sajad Azizi

Copyright ( 2019 Qianqian Fang et al. This is an open access article distributed under the Creative Commons Attribution License, which permits unrestricted use, distribution, and reproduction in any medium, provided the original work is properly cited.

In this paper, a new modular upper limb rehabilitation exoskeleton, which is actuated by a parallel mechanical structure, is designed to help stroke patients. For analysing the relationship between motor torque and joint torque of the novel exoskeleton, a conversion algorithm mapping motor motion to joint motion is developed here. Then, to simplify the dynamics model of exoskeleton with parallel actuated joints, the serial equivalence configuration dynamics of the exoskeleton is established to be equivalent to the parallel joints dynamics. Afterwards, a torque controller used for our exoskeleton is designed based on the proposed conversion algorithm and the inverse dynamics of exoskeleton. It should be noted that the controller mentioned above combines both conversion algorithm and joint position decoupling. At last, for verifying the effectiveness of the proposed algorithms, a trajectory tracking simulation is given, and the simulated results show the proposed algorithms are valid.

\section{Introduction}

Stroke is a neurological disease with a high prevalence and often leads to disability. One major symptom after stoke is the decrease in the function of patients' upper extremities, which seriously affects their daily life [1]. The most effective therapy plan is the rehabilitation training for stroke patients [2]. However, the traditional treatment process is carried out by professional therapists, which is time-consuming and expensive.

In order to solve the defects of hand-assisted rehabilitation process, many upper limb exoskeleton systems were developed for helping patients. A different flexible upper limb exoskeleton is introduced in [3-5]. More knowledge about flexible joints can be found in [6, 7]. A fivedegree-of-freedom (DOF) upper limb exoskeleton is developed in [8], which is driven by using DC motors and pneumatic muscles. A 5-DOF exoskeleton is developed by Mao and Agrawal [9], which is based on three light weight cuffs. These exoskeletons are of traditional serial structure with enough workspace. In addition, it is easy to control them. However, compared with the parallel mechanism [10], the series structure is with low precision and it has the disadvantage of small stiffness. Each degree of freedom of the serial mechanism is driven by a single driving mechanism, which causes the serial mechanism to have lower rigidity than the parallel mechanism. It also leads the bearing capacity of the series mechanism to be weaker than that of the parallel mechanism. Klein et al. [11] designed a pneumaticdriven upper limb rehabilitation exoskeleton with parallel structure. In addition, Takaiwa et al. [12] designed a set of rehabilitation device by using a parallel robotic arm pneumatically actuated in the wrist. Tsagarakis and Caldwell [13] designed a 7-DOF exoskeleton robot by using pneumatic muscle actuators as the power source. Although pneumatic actuators require less maintenance, they are less accurate [14]. In [9], Mao and Agrawal designed a 5-DOF cabledriven arm exoskeleton (CAREX) with the motors need to be placed separately on an aluminum frame. In [15], Xiao et al. designed a cable-driven differential structure for the 
upper limb exoskeleton with the motors mounted on the backboard. However, the separate fixation of the exoskeleton motors makes the overall structure of the exoskeleton more complex and less compact. In this paper, we designed a 6DOF modular cable-driven upper limb rehabilitation exoskeleton robot with parallel actuated joints based on human anatomy and biological principles to overcome the above limitations. The overall structure of the exoskeleton can be moved and fixed on any bracket which makes it compact and simple. The exoskeleton consists of three independent modules representing three joints of the human upper limb, and each module achieves two DOFs of the human upper limb. The structure can contribute to both a single joint and multijoint rehabilitation training for patients. In addition, it can also be used to train the patient's left arm and right arm.

The main control strategies in the upper limb rehabilitation exoskeleton are passive control [16]. Passive control means that patients follow the movement of exoskeleton along a desired reference trajectory $[17,18]$. The basic and simple passive technique is PID feedback control which often cannot obtain precise control results [19]. In addition, some more complex approaches, such as neural network PID [20], sliding mode control [21], and fuzzy logic techniques [22], have been applied to upper limb rehabilitation exoskeleton control. However, these algorithms are usually complicated and difficult to be implemented in practical applications. With the fast development of hardware system, EMG methods [23, 24] are applied to upper limb exoskeleton, which measure muscle activity by surface EMG to directly detecting motional intention of patients. But these methods need expensive measuring equipment. Recently, some intelligent control methods [25], such as learning techniques [26, 27], are tried, but they need expensive calculation efforts. There are also some other methods performed by calculating inverse kinematics of exoskeleton to implement accurate position and trajectory planning [28]. However, these methods are only for series exoskeleton configurations. In order to achieve friendly human-exoskeleton interaction, some force control methods [29] are used, such as the admittance control [30]. Unfortunately, these methods do not establish and combine the dynamics model of exoskeletons. In addition, some adaptive robust control algorithms [31] are also applied in exoskeleton control, but the adaptive parameters are usually hard to be tuned and identified. Although some force control algorithms, such as the impedance control [32], establish the dynamics model of the exoskeleton and apply them to the control, these methods are usually used for series exoskeleton and cannot be applied directly to our exoskeleton with parallel actuated joints.

Therefore, for obtaining accurate motion control and good dynamic performance of our parallel exoskeleton, we establish the dynamics model of exoskeleton by simplifying it as a serial equivalence configuration firstly in this paper. Then, a conversion algorithm is proposed to map the motor motion to the joint motion. Afterwards, a torque control method combined with the conversion algorithm and position decoupling is designed for our exoskeleton. For verifying the effectiveness of the proposed algorithms, a polynomial trajectory with fifth interpolation is simulated. Finally, the simulation results prove the validity of the proposed algorithms. The main contributions of this paper are as follows: (1) By converting motion relationship between motors and joints of the designed exoskeleton, the parallel exoskeleton is equivalently simplified to a serial exoskeleton to establish inverse dynamics; (2) a novel parallel upper limb rehabilitation exoskeleton with modular structure was designed; (3) a trajectory tracking control method for the exoskeleton was designed based on the proposed conversion algorithm of equivalent simplified inverse dynamics.

The paper is organized as follows: Section 2 is the mechanical design of the 6-DOF upper limb rehabilitation exoskeleton based on parallel actuated joints. Section 3 introduces conversion algorithm which maps motor motion to joint motion. Section 4 discusses the torque controller design, system stability analysis, and trajectory tracking simulation results. Section 5 discusses the simulation results. At last, conclusion is shown in Section 6.

\section{Cable-Actuated Parallel Exoskeleton Design}

The exoskeleton robot shown in Figure 1 for rehabilitation training of upper limb uses the cable-actuated parallel mechanism to achieve 6-DOF movement of the human upper limb. We approximated the shoulder as a spherical joint, the elbow as a rotary joint, and the wrist as a 2-DOF nonspherical joint.

In the mechanical design, each joint exists as an independent module, so the exoskeleton has three modules representing the three joints of the human upper limb, that is, the shoulder joint, the elbow joint, and the wrist joint. Each module is a differential mechanism and has two coupled motors to achieve two DOFs of the human upper limb. The shoulder joint module achieves shoulder abduction/adduction freedom and flexion/extension freedom. The elbow joint module achieves shoulder internal/external rotation and elbow flexion/extension. The wrist joint module achieves forearm supination/pronation and wrist flexion/ extension.

The three modules have the same structural design principle, so the shoulder joint module show in Figure 2 is used as an example to analyse joint motion. $S$ is the shoulder joint rotation center. The main driving mechanism consists of two small straight wheels (SSW1 and SSW2), two large straight circles (SSC1 and SSC2), two second-order shafts (SSO1 and SSO2), and two small wheels (SW1 and SW2) each with a second-order shaft.

Take the one-sided driving mechanism as an example. When motor 1 actuates the small straight wheel SSW 1 to rotate, the large straight circle SSC1 will also rotate by the actuation of SSW1. SSC1 and the second-order shaft SSO1 are integrated structures, so the small straight wheel SW1 can be driven by using SSO1 to achieve the rotation of the exoskeleton. Specifically, SSO1 and the second-order shaft on SW1 are driven with a pair of wire ropes represented by the green line and red line shown in Figure 2(b). When SSO1 rotates, one of the two wire ropes that are entangled in SSO1 

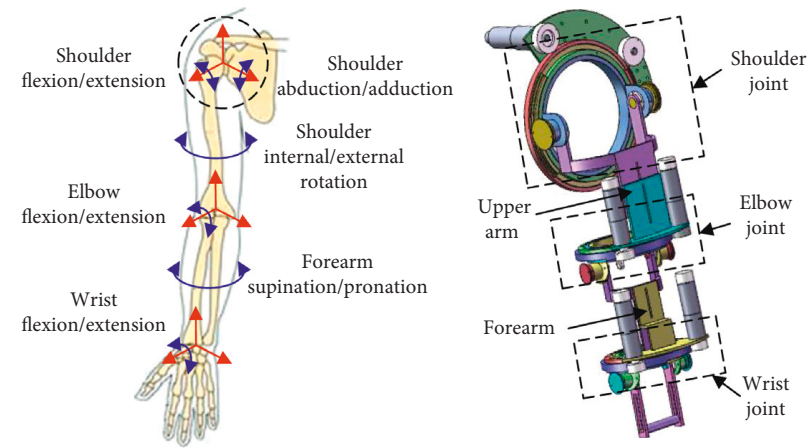

Figure 1: Model of the upper limb exoskeleton.

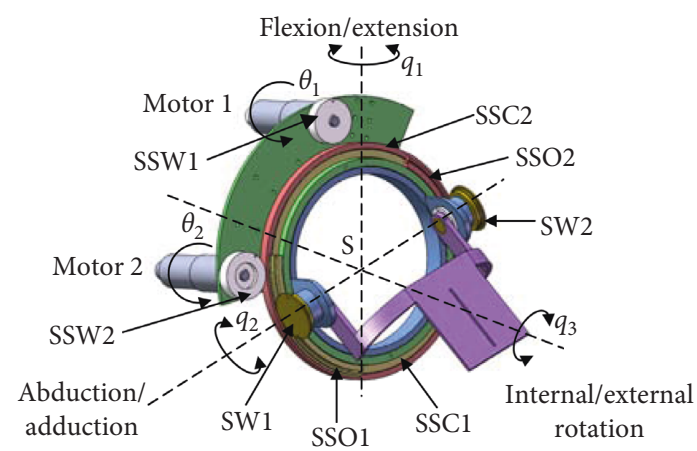

(a)

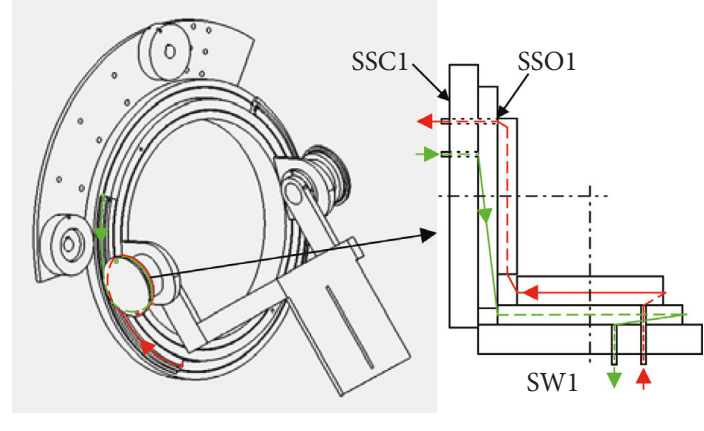

(b)

Figure 2: The shoulder joint module. (a) The schematic diagram. (b) The actual way of installing the wire ropes.

is released to wrap around the corresponding shaft of SW1. At the same time, the other of the two wire ropes is released from SW1 to wound on the corresponding shaft of SSO1. By using this method, we can achieve the corresponding rotation between SSO1 and SW1. Finally, since the upper arm is attached to the small wheel SW1, the small wheel rotation drives the upper arm rotation.

When the two motors are at the same speed with the same rotation direction, the exoskeleton can achieve the flexion/extension of the shoulder joint. When the two motors are at the same speed with the opposite rotation direction, the exoskeleton can achieve the abduction/adduction movement of the shoulder joint. When the rotation speed of motor 1 is faster than that of motor 2, then the exoskeleton can achieve the abduction and flexion or adduction and extension of the shoulder joint. When the rotation speed of motor 2 is faster than that of motor 1 , then the exoskeleton can achieve the abduction and extension or adduction and flexion movement of the shoulder joint. For more structural design details, one can refer to our previous work $[33,34]$.

\section{The Proposed Conversion Algorithm}

Each module is a differential mechanism, and therefore, the coupled relationship exists between two degrees of freedom for each joint. If a set of desired exoskeleton angular velocities are known, the required motor angular velocities can be obtained by

$$
\dot{q}=\frac{\mathrm{d} q}{\mathrm{~d} t}=J \dot{\theta}
$$

where $q \in \mathfrak{R}^{6 \times 1}$ is the generalized coordinate which can determine the position of joints of the exoskeleton, $\theta \in \mathfrak{R}^{6 \times 1}$ is the generalized coordinate associated with the position of motors, and $J \in \mathfrak{R}^{6 \times 6}$ is the Jacobian matrix relating motor velocities to the exoskeleton joint velocities.

The transposition of the Jacobian matrix correlates the torque exerted on the joint with the torque of the actuator, so we can get the torque of the motor by the following equation:

$$
\tau_{\theta}=J^{\mathrm{T}} \tau,
$$

where $\tau_{\theta} \in \Re^{6 \times 1}$ is the torque generated by the motors, $\tau \in \mathfrak{R}^{6 \times 1}$ is the motor driving torque mapped to the load side, and $J^{\mathrm{T}}$ is the transpose of Jacobian matrix $J$.

By mapping motor motion to joint motion, the exoskeleton with parallel actuated joints can be equivalent to a 6-DOF serial robotic arm. Therefore, the dynamics of a 6DOF serial exoskeleton structure is the same as that of a 6link rigid manipulator. The dynamic modelling methods commonly used for a serial robot are the Newton-Euler iteration method and the Lagrangian method. Since iteration efficiency of Newton-Euler is very high [35], this paper uses the Newton-Euler iteration method to carry out dynamic modelling of the simplified exoskeleton. The torque balance equation of the system can be obtained according to the torque of the motor end as follows: 


$$
\begin{aligned}
\tau_{\theta} & =I_{\theta} \ddot{\theta}+\tau_{f, m}+J^{\mathrm{T}} \tau_{q}, \\
\tau_{f, m} & =b \dot{\theta}+f_{c},
\end{aligned}
$$

where $I_{\theta}=\operatorname{diag}\left\{B_{i}\right\} \in \mathfrak{R}^{6 \times 6}$ is the inertia of the motor rotor, $\tau_{f, m}$ is the friction torque at the connecting rod end which is equivalent to the end of the connecting rod by viscous friction and Coulomb friction at the motor end, $\tau_{q}$ is the joint torque, $b$ is the viscous friction coefficient of the motor side, and $f_{c}$ is the Coulomb friction at the motor side.

By analysing the movement at the joint end, the equation of dynamics can be written as follows:

$$
\tau_{q}=M(q) \ddot{q}+C(q, \dot{q}) \dot{q}+G(q)
$$

where $M(q) \in \mathfrak{R}^{6 \times 6}$ is the symmetric and positive-definite inertia matrix, $C(q, \dot{q}) \in \mathfrak{R}^{6 \times 6}$ is the centripetal and Coriolis matrix, and $G(q) \in \mathfrak{R}^{6 \times 1}$ is the gravity vector.

Equations (3) and (5) can be combined to obtain the dynamic state equation:

$$
H(q) \ddot{q}+C(q, \dot{q}) \dot{q}+G(q)=\tau-J^{-T} \tau_{f, m},
$$

where $H(q)=M(q)+J^{-T} I_{\theta} J^{-1}$.

In the shoulder joint, the reduction ratios from the output shaft of the motor 1 reducer and motor 2 reducer to the large straight circle corresponding to those motors are $i_{1}$ and $i_{2}$, respectively. And $c_{1}$ and $c_{2}$ represent the reduction ratios from large straight circle to small wheel corresponding to that large straight circle, respectively. In the mechanical design, we let $c_{1}=c_{2}$. By decoupling analysis and calculating the shoulder joint, we can get the following equation:

$$
\left[\begin{array}{l}
\dot{q}_{1} \\
\dot{q}_{2}
\end{array}\right]=\left[\begin{array}{cc}
\frac{1}{2 i_{1}} & \frac{1}{2 i_{2}} \\
-\frac{1}{i_{1}\left(c_{1}+c_{2}\right)} & \frac{1}{i_{2}\left(c_{1}+c_{2}\right)}
\end{array}\right]\left[\begin{array}{l}
\dot{\theta}_{1} \\
\dot{\theta}_{2}
\end{array}\right]=J_{S}\left[\begin{array}{l}
\dot{\theta}_{1} \\
\dot{\theta}_{2}
\end{array}\right],
$$

where $J_{S} \in \mathfrak{R}^{2 \times 2}$ is the Jacobian matrix in the shoulder joint.

Similarly, for the elbow and wrist joints, we can get the following equations:

$$
\begin{aligned}
& {\left[\begin{array}{l}
\dot{q}_{3} \\
\dot{q}_{4}
\end{array}\right]=J_{E}\left[\begin{array}{c}
\dot{\theta}_{3} \\
\dot{\theta}_{4}
\end{array}\right],} \\
& {\left[\begin{array}{c}
\dot{q}_{5} \\
\dot{q}_{6}
\end{array}\right]=J_{W}\left[\begin{array}{c}
\dot{\theta}_{5} \\
\dot{\theta}
\end{array}\right],}
\end{aligned}
$$

where $J_{E} \in \mathfrak{R}^{2 \times 2}$ and $J_{W} \in \mathfrak{R}^{2 \times 2}$ are the Jacobian matrix in the elbow and wrist joints, respectively.

Then, the Jacobian matrix $J$ can be obtained by

$$
J=\left[\begin{array}{ccc}
J_{S} & & \\
& J_{E} & \\
& & J_{W}
\end{array}\right] .
$$

Finally, for a 6-DOF exoskeleton with parallel-driven joints, giving the desired joint position, velocity, and acceleration, the motor torque can be calculated based on the aforesaid results. More similar modelling details can be found in $[11,36]$.

\section{Trajectory Tracking Control and Simulations}

4.1. System Stability Analysis. In order to better observe the driving torque of each motor of the exoskeleton after mapping motor motion to joint motion, this section designs a controller shown in Figure 3 based on joint angle decoupling introduced in detail in our previous paper [34] and conversion algorithm with the computational torque method for parallely connected exoskeleton robots and performs trajectory tracking control simulation based on the planned motor trajectory.

The control law of the controller we designed is shown below:

$$
\tau=H(q)\left[k_{p} e+k_{d} \dot{e}+\ddot{q}_{d}\right]+C(q, \dot{q}) \dot{q}+G(q)+J^{-T} \tau_{f, m},
$$

where $e=q_{d}-q, \dot{e}=\dot{q}_{d}-\dot{q}$, and $\ddot{e}=\ddot{q}_{d}-\ddot{q}$ are the position error, velocity error, and acceleration error, respectively.

After equation (10) is incorporated into equation (6), we can obtain the following equation:

$$
H(q) \ddot{q}=H(q)\left[k_{p} e+k_{d} \dot{e}+\ddot{q}_{d}\right] .
$$

Since $H(q)$ is the same symmetric and positive-definite matrix as $M(q)$ [35], $H(q)$ is invertible. For this reason, equation (11) can be simplified as

$$
\ddot{q}=k_{p} e+k_{d} \dot{e}+\ddot{q}_{d} .
$$

Substituting $\ddot{e}=\ddot{q}_{d}-\ddot{q}$ into equation (12), we can get the following equation:

$$
\ddot{e}+k_{d} \dot{e}+k_{p} e=0 .
$$

The Lyapunov function is designed as [31]

$$
V=\frac{\left(\dot{e}^{T} \dot{e}\right)}{2}+\frac{\left(e^{T} k_{p} e\right)}{2}
$$

Then, the time derivative of the Lyapunov function can be deduced as follows:

$$
\dot{V}=\frac{\left(\ddot{e}^{T} \dot{e}+\dot{e}^{T} \ddot{e}\right)}{2}+\frac{\left(\dot{e}^{T} k_{p} e+e^{T} k_{p} \dot{e}\right)}{2} .
$$

Since $\ddot{e}^{T} \dot{e}=\dot{e}^{T} \ddot{e}$ and $\dot{e}^{T} k_{p} e=e^{T} k_{p} \dot{e}$, equation (15) can be rewritten as

$$
\dot{V}=\dot{e}^{T} \ddot{e}+\dot{e}^{T} k_{p} e .
$$

Combined with equation (13), equation (16) becomes

$$
\dot{V}=\dot{e}^{T}\left(\ddot{e}+k_{p} e\right)=-\dot{e}^{T} k_{d} \dot{e} \leq 0 .
$$

Therefore, it can be known that $(e, \dot{e})=(0,0)$ is the global asymptotically stable equilibrium point of the exoskeleton system from the LaSalle theorem. In addition, more similar robot controller stability analyses can also be found in $[31,37,38]$. 


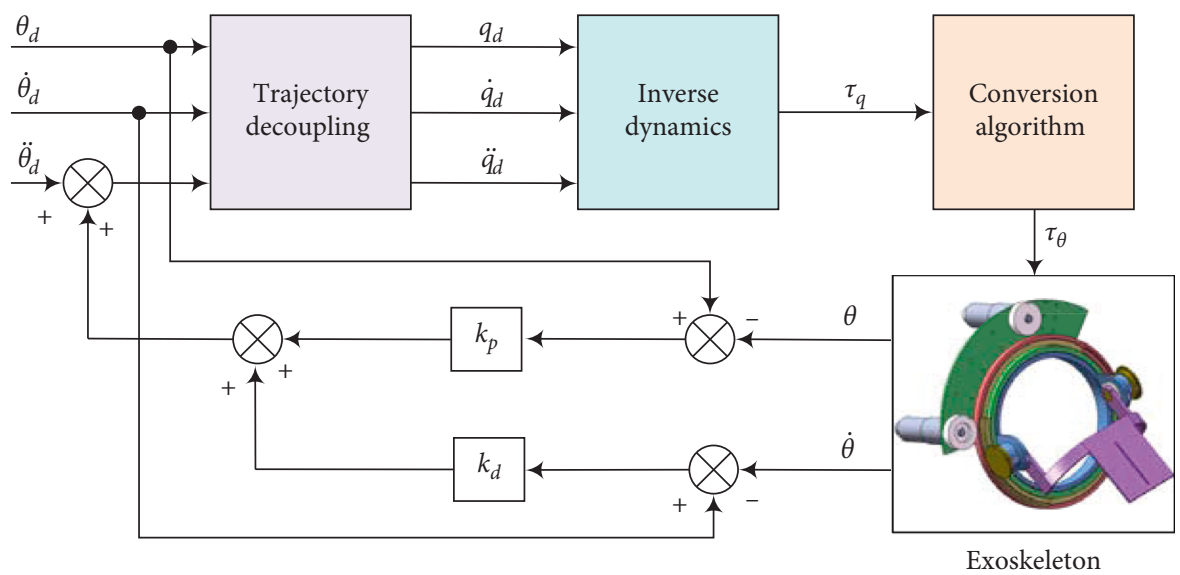

FIgURE 3: The controller based on joint angle decoupling and conversion algorithm. $\theta_{d}, \dot{\theta}_{d}$, and $\ddot{\theta}_{d}$ represent the position, velocity, and acceleration of the desired motor trajectory of the exoskeleton. $q_{d}, \dot{q}_{d}$, and $\ddot{q}_{d}$ represent the position, velocity, and acceleration of the desired joint trajectory of the exoskeleton. $k_{p}$ and $k_{d}$ are the diagonal positive-definite control gain matrices.

4.2. Simulation and Analysis. In the controller, the desired motor trajectory of the exoskeleton is planned. Then desired joint trajectory of the simplified serial robot is calculated according to the trajectory decoupling algorithm. By calculating the inverse dynamics of the serial robot, each joint moment is obtained. Therefore, the actual driving torque of each motor can be obtained by using the proposed conversion algorithm, resulting in the movement of the upper limb rehabilitation exoskeleton. It should be noted that the unmodelled errors and disturbances in robot dynamics are suppressed by the feedback parts of the controller, i.e., $k_{p}$ and $k_{d}$. With regulation of the control gains $k_{p}$ and $k_{d}$, the joint motor can follow the desired trajectory for motion. Each motor trajectory planned in this paper is based on fifthorder polynomial as follow:

$$
\theta(t)=a_{0}+a_{1} t+a_{2} t^{2}+a_{3} t^{3}+a_{4} t^{4}+a_{5} t^{5} .
$$

The fifth-order polynomial can minimize the jerk of the motion trajectory. The researchers have found that normal human movement follows a trajectory that minimizes total movement jerk based on observations of voluntary motion [39]. It is expected that the exoskeleton will move along a trajectory similar to that of a normal human body to ensure its comfort and fitness for rehabilitation. And the use of polynomials has great advantages in real-time applications, especially in rehabilitation. The control of the human trajectory can be enhanced by the ability of replanning coefficients of polynomials or superimposing a new trajectory based on the previous one in real time by using this method. And, the angular velocity and angular acceleration of the fifth-order curve are both zero at the initial point and the end point. The coefficients of the polynomial can be obtained as follows: $a_{i(i=1, \ldots, 6)}=[0,0,0,0.1257,-0.0377,0.003]$.

For verifying the performance of the algorithm proposed above, elbow flexion/extension motion is simulated below. At the initial moment, the exoskeleton flexion angle is zero; that is, from the visual point of view, the exoskeleton arm naturally hangs down, and the three joints of the arm form a straight line perpendicular to the earth. Then the flexion of the elbow joint is performed, during which the shoulder joint and upper arm remained stationary, and the exoskeleton arm is stopped at the position where the flexion angle of the elbow joint is $90^{\circ}$.

Figure 4 shows the actual motor torque curve obtained by the proposed conversion algorithm. In the elbow joint, there are two situations that need to be noticed; that is, when the two motors are at the same speed with the same rotation direction, the exoskeleton can achieve the internal/external rotation movement of the upper arm; when the two motors are at the same speed with the opposite rotation direction, the exoskeleton can achieve the flexion/extension of the elbow joint. Therefore, as the angle of elbow flexion increases, the more gravity the elbow joint needs to overcome, and the torque of two motors of the elbow gradually increases in the opposite direction.

Since only elbow flexion/extension movement are performed, the trajectory tracking effect shown in Figure 5 of motor 3 and motor 4 at the elbow joint is analysed in this paper. It is assumed that the initial positions of both motors at the initial time are zero. As shown in Figure 5, the angles of two motors have the same tendency to move in the opposite directions which corresponds to the elbow joint torque obtained above. Position error and root-meansquared error (RMSE) of elbow joint motors are shown in Figure 6 and Table 1. The error between the expected position and the actual position of each motor is almost zero, which meets the purpose of rehabilitation training of the exoskeleton and shows the proposed algorithms are valid.

The actual motor torque curve of simultaneous motion of multiple joints is shown in Figure 7. In Figure 7, in the shoulder joint, an abduction movement is performed from $0^{\circ}$ to $90^{\circ}$. Simultaneously, in the elbow joint, flexion movement is performed from $0^{\circ}$ to $90^{\circ}$. When the shoulder joint and the elbow joint move synchronously, the maximum driving torque of motor 1 to motor 4 is $0.1206 \mathrm{Nm},-0.1205 \mathrm{Nm}$, $-0.0225 \mathrm{Nm}$, and $0.016 \mathrm{Nm}$, respectively.

In order to observe the effect of the disturbance, perturbation force of $5 \mathrm{Nm}$ is applied to each joint of the exoskeleton at $t=2 \mathrm{~s}$ on the basis of simultaneous movement 


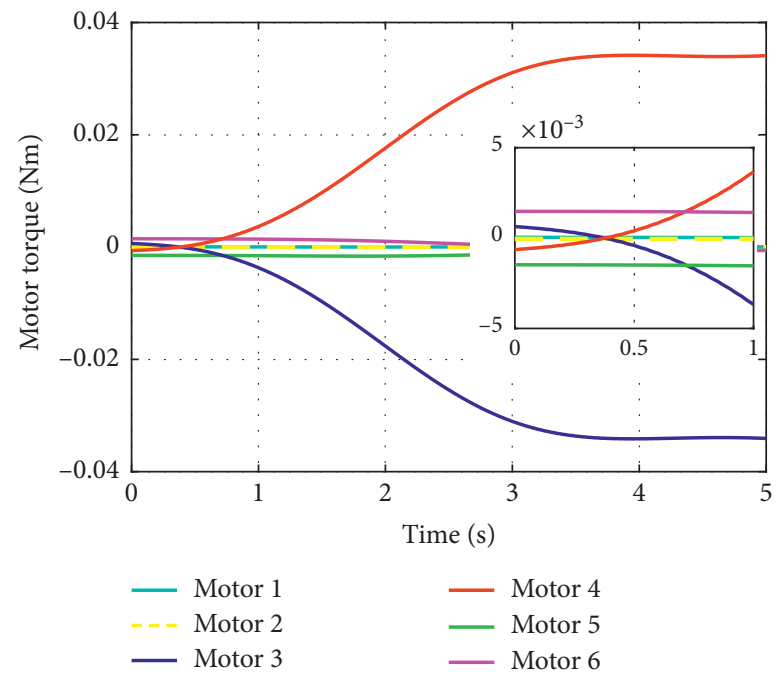

FIgURE 4: The actual motor torque curve by decoupling joint torque.

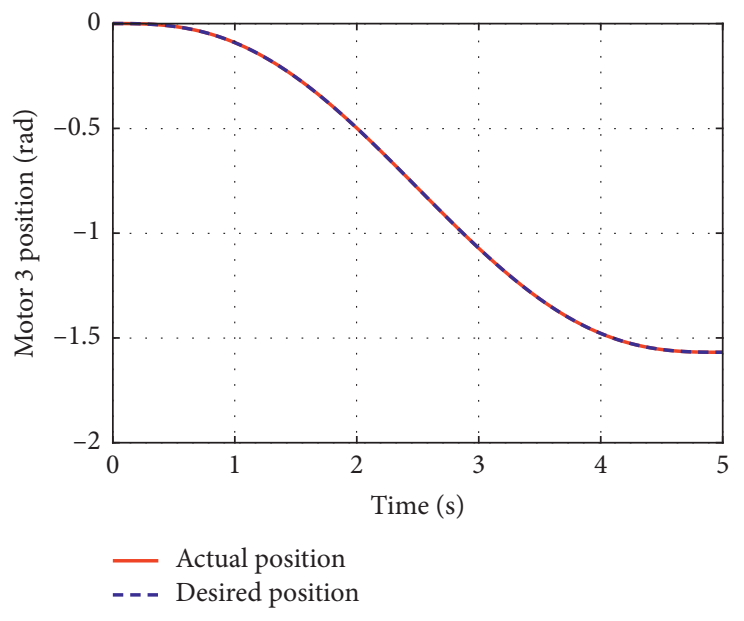

(a)

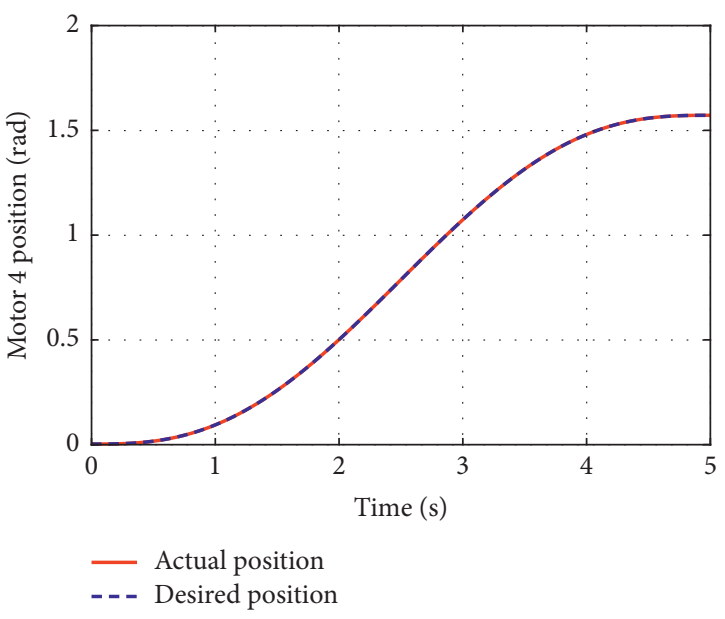

(b)

Figure 5: Trajectory tracking of elbow joint motors. (a) Motor 3. (b) Motor 4.

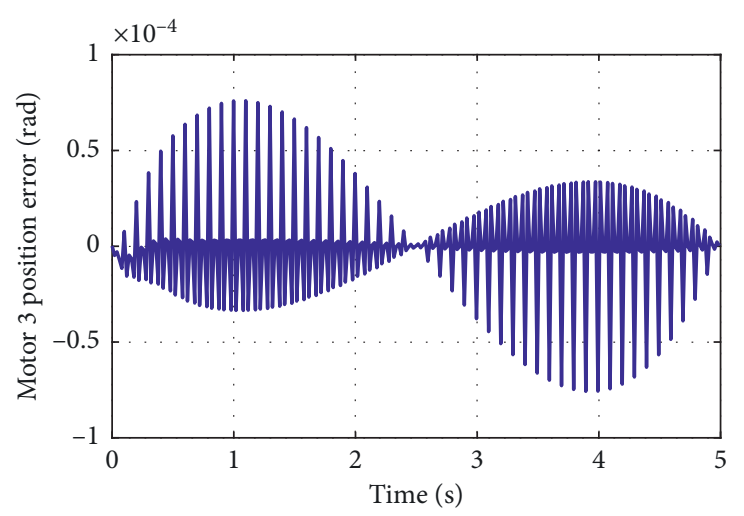

Motor 3

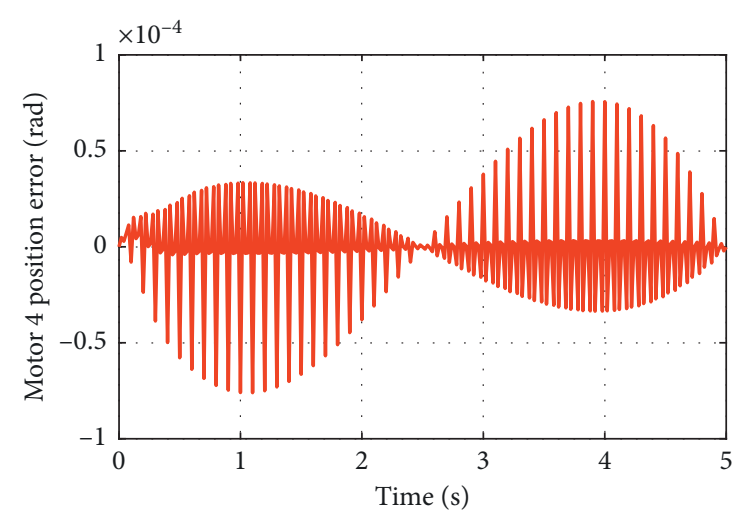

Motor 4

(a)

(b)

Figure 6: Position errors of elbow joint motors. (a) Motor 3. (b) Motor 4. 
TABLE 1: Root-mean-squared error of position tracking.

\begin{tabular}{lc}
\hline Motor no. & Errors/RMSE (rad) \\
\hline 3 & $2.87 e-05$ \\
4 & $2.87 e-05$ \\
\hline
\end{tabular}

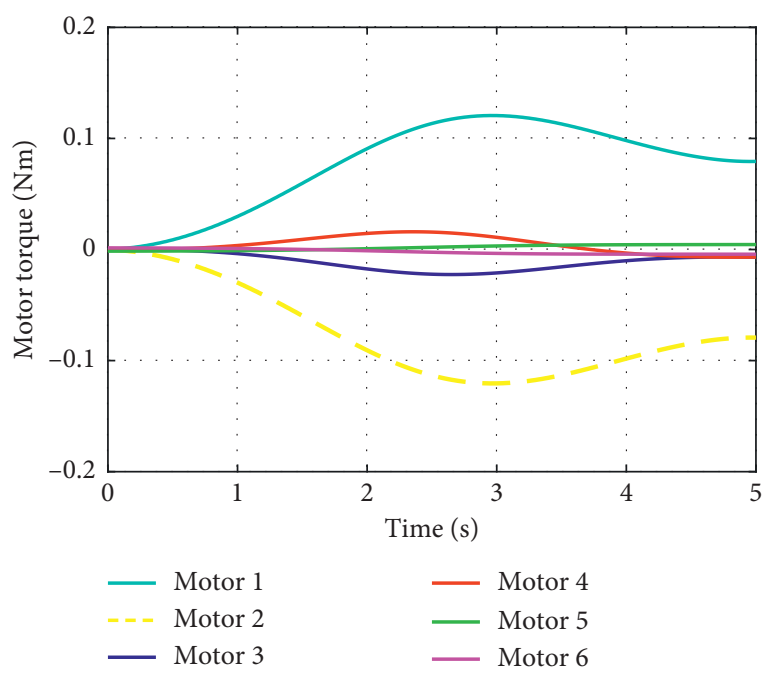

FIGURE 7: The actual motor torque curve of simultaneous motion of multiple joints.

of the shoulder joint and the elbow joint. After adding the disturbance, the actual motor torque curve is shown in Figure 8. The trajectory tracking effect and RMSE of the shoulder joint and elbow joint motors are shown in Figure 9 and Table 2. Once the disturbance is present, the torque of the motor fluctuates. The actual trajectory also deviates from the expected trajectory. The maximum position tracking RMSE of motors reached $0.6696 \mathrm{rad}$, and the minimum position tracking RMSE of motors reached $0.0795 \mathrm{rad}$.

\section{Discussion}

In this paper, a novel upper limb rehabilitation exoskeleton is developed for stroke patients which is cable-driven and is serially composed of three parallel joints modules. In addition, a conversion algorithm is proposed to solve the relationship between motor torque and joint torque of the novel exoskeleton, which is also used to simplify the dynamics model of exoskeleton with parallel actuated joints. Because the dynamics of the parallel exoskeleton robots are difficult to be modelled, the serial equivalence configuration dynamics of the exoskeleton are established to be equivalent to the parallel joints dynamics. Afterwards, a torque controller used for our exoskeleton is designed based on the proposed conversion algorithm, and the system stability is also analysed. In order to verify the effectiveness of the proposed algorithms, a trajectory tracking simulation is given. As shown in Figure 4, it can be found that the torque of motors 3 and 4 is large and reverse because the simulated bending and extending motion of the elbow joint is driven and constructed by the reverse motions of the two motors. The maximum driving torque of motor 3 and motor 4 is

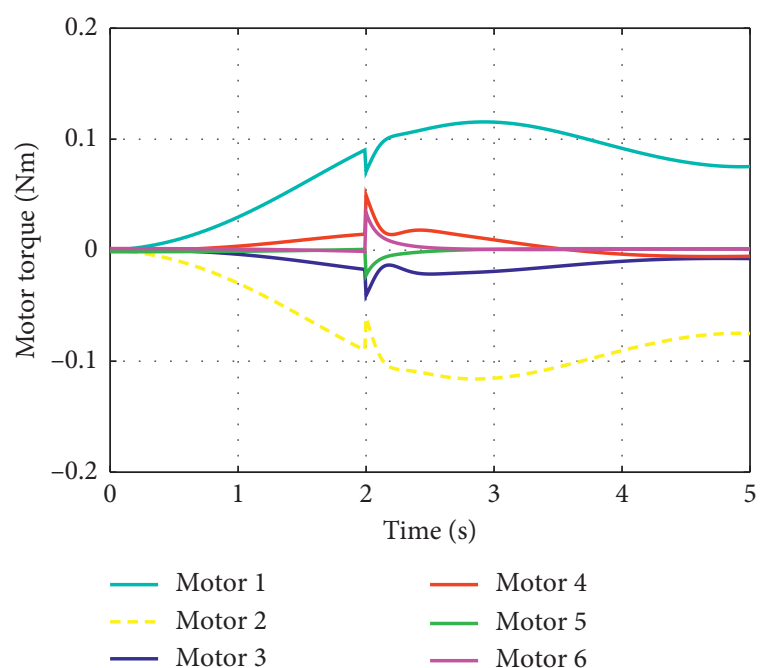

Figure 8: Actual motor torque curve with disturbance.

TABLE 2: Root-mean-squared error of position tracking with disturbance.

\begin{tabular}{lc}
\hline Motor no. & Errors/RMSE (rad) \\
\hline 1 & 0.0795 \\
2 & 0.1004 \\
3 & 0.1971 \\
4 & 0.6696 \\
\hline
\end{tabular}

$-0.0342 \mathrm{Nm}$ and $0.0342 \mathrm{Nm}$, respectively. In addition, the torque of other motors is small and not zero because the friction model is considered and established in the simulation. From Figure 5, the results show that the actual position of motor 3 and motor 4 can track the desired position well by using the proposed trajectory tracking method for the novel exoskeleton. In addition, it can be known that the position tracking errors of motors are limited to $2.87 \times 10^{-5} \mathrm{rad}$ as shown in Figure 6. So, it can be concluded that the developed trajectory tracking controller based on the parallel joint exoskeleton dynamics can contribute to the trajectory tracking control very well, and the position tracking errors are small in this method. Therefore, the results show that the proposed algorithms are effective and correct. In the future work, the experiments of the proposed algorithms will be conducted in the designed exoskeleton robot platform.

\section{Conclusion}

A newly designed modular upper limb rehabilitation exoskeleton with parallel actuated joints is described in this paper. The three modules represent the three joints of the human upper limb, which can achieve rehabilitation training for any joint of the left arm or right arm of the human upper limb. To obtain the relationship between motor torque and joint torque of exoskeleton, a decoupling Jacobian matrix is calculated to map the joint torque to the motor torque. Then, for solving the joint torque, a dynamics model of a serial equivalence configuration of 


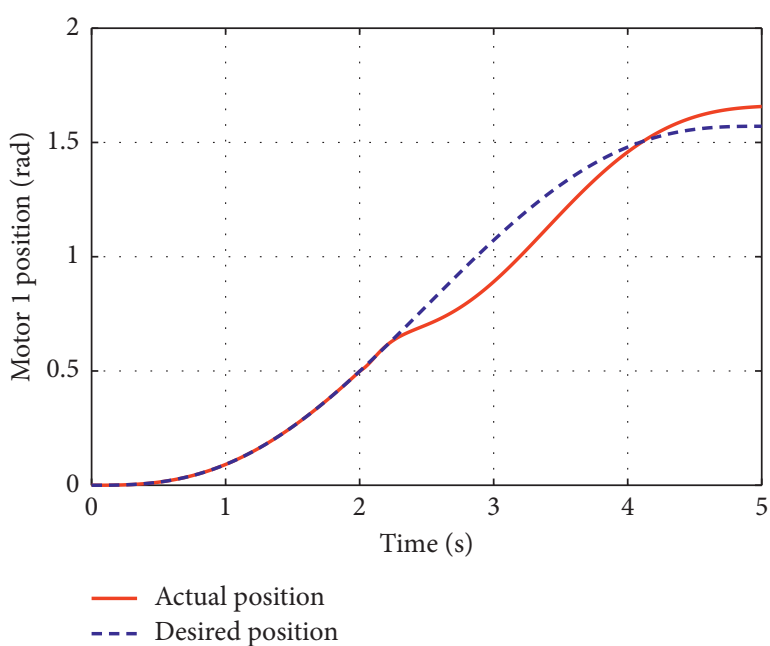

(a)

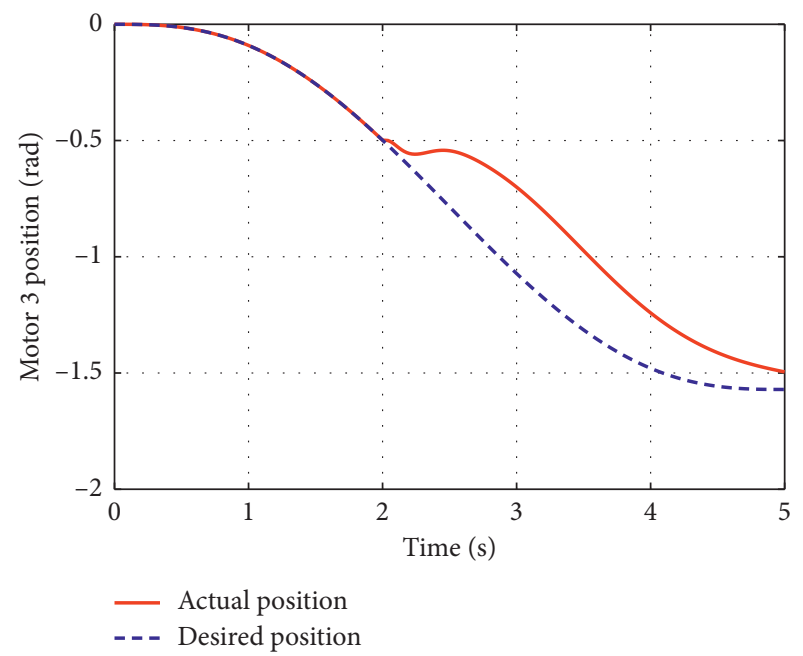

(c)

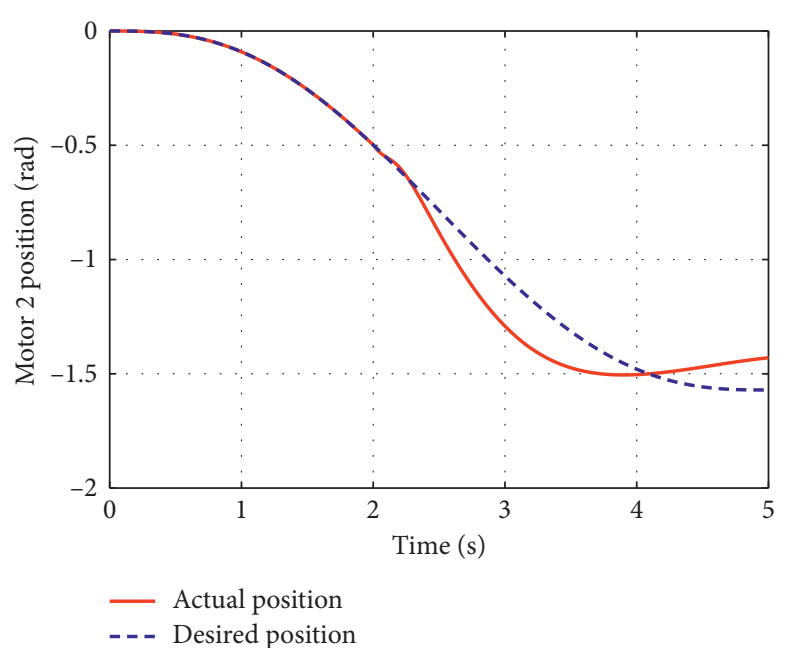

(b)

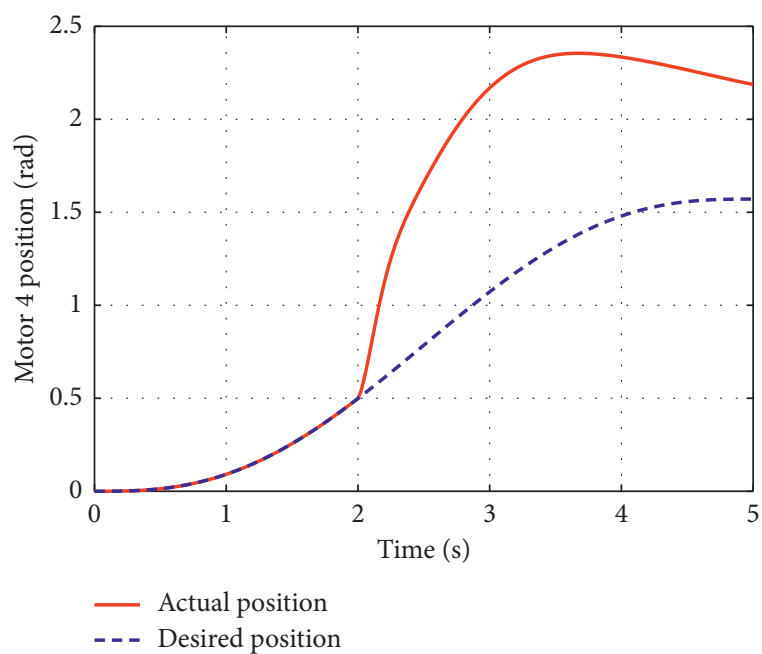

(d)

Figure 9: Trajectory tracking of motors with disturbance. (a) Motor 1. (b) Motor 2. (c) Motor 3. (d) Motor 4.

the exoskeleton is established. Afterwards, a torque controller used to control our exoskeleton is designed, which especially contains the proposed conversion algorithm and joint position decoupling. The system stability is also analysed. For proving the validity of the proposed algorithms, a trajectory tracking control is simulated. From the simulation results, we find the trajectory tracking performance is good, which shows the proposed algorithms are valid.

\section{Data Availability}

As the project will not be finished until July 2020 and we have confidentiality agreement with State Key State Key Laboratory of Robotics and System, the data could not be released so far. For any information about the article, please contact us via yhzhu@hit.edu.cn.

\section{Conflicts of Interest}

The authors declare that they have no conflicts of interest.

\section{Acknowledgments}

This work was supported by the National Key R\&D Program of China (Grant no. 2017YFB1302301) and the Joint Research Fund (Grant nos. U1613218, U1713201, and U1613219) of the National Nature Science Foundation of China (NSFC) and Shenzhen.

\section{References}

[1] K. D. Fitle, A. U. Pehlivan, and M. K. O'Malley, "A robotic exoskeleton for rehabilitation and assessment of the upper limb following incomplete spinal cord injury," in Proceedings of the IEEE International Conference on Robotics and Automation, pp. 4960-4966, IEEE, Seattle, WA, USA, May 2015.

[2] S. M. Hatem, G. Saussez, M. della Faille et al., "Rehabilitation of motor function after stroke: a multiple systematic review focused on techniques to stimulate upper extremity recovery," Frontiers in Human Neuroscience, vol. 10, 2016.

[3] J. C. Perry, J. Rosen, and S. Burns, "Upper-limb powered exoskeleton design," IEEE/ASME Transactions on Mechatronics, vol. 12, no. 4, pp. 408-417, 2007. 
[4] N. Li, T. Yang, P. Yu et al., "Bio-inspired upper limb soft exoskeleton to reduce stroke-induced complications," Bioinspiration \& Biomimetics, vol. 13, no. 6, Article ID 066001, 2018.

[5] X. Jin, S. Zhu, X. Zhang, X. Zhu, and Z. Pan, "Structure design and experiment of knee joint of hydraulic driven lower-limb power-assistance exoskeleton robot," Transactions of the Chinese Society of Agricultural Engineering, vol. 33, no. 5, pp. 26-31, 2017.

[6] W. He and S. Zhang, "Control design for nonlinear flexible wings of a robotic aircraft," IEEE Transactions on Control Systems Technology, vol. 25, no. 1, pp. 351-357, 2017.

[7] A. De Luca and W. J. Book, "Robots with flexible elements," in Handbook of Robotics, pp. 243-282, Springer, Cham, Switzerland, 2016.

[8] F. Martinez, I. Retolaza, A. Pujana-Arrese, A. Cenitagoya, J. Basurko, and J. Landaluze, "Design of a five actuated DoF upper limb exoskeleton oriented to workplace help," in Proceedings of the IEEE Ras \& Embs International Conference on Biomedical Robotics and Biomechatronics. IEEE, pp. 169174, Scottsdale, AZ, USA, October 2008.

[9] Y. Mao and S. K. Agrawal, "Design of a cable-driven arm exoskeleton (CAREX) for neural rehabilitation," IEEE Transactions on Robotics, vol. 28, no. 4, pp. 922-931, 2012.

[10] A. Gupta and M. K. O’Malley, "Design of a haptic arm exoskeleton for training and rehabilitation," IEEE/ASME Transactions on Mechatronics, vol. 11, no. 3, pp. 280-289, 2006.

[11] J. Klein, S. J. Spencer, J. Allington et al., "Biomimetic orthosis for the neurorehabilitation of the elbow and shoulder (BONES)," in Proceedings of the 2008 2nd IEEE RAS \& EMBS International Conference on Biomedical Robotics and Biomechatronics, IEEE, Scottsdale, AZ, USA, October 2008.

[12] M. Takaiwa and T. Noritsugu, "Development of wrist rehabilitation device using pneumatic parallel manipulator energy saving and new application on fluid power," in Proceedings of the First China-Japan Joint Workshop on Fluid Power, pp. 2302-2307, Lanzhou, China, August 2010.

[13] N. G. Tsagarakis and D. G. Caldwell, "Development and control of a "soft-actuated" exoskeleton for use in physiotherapy and training," Autonomous Robots, vol. 15, no. 1, pp. 21-33, 2003.

[14] R. A. R. C. Gopura, D. S. V. Bandara, K. Kiguchi, and G. K. I. Mann, "Developments in hardware systems of active upper-limb exoskeleton robots: a review," Robotics and Autonomous Systems, vol. 75, pp. 203-220, 2015.

[15] F. Xiao, Y. Gao, Y. Wang, Y. Zhu, and J. Zhao, "Design and evaluation of a 7-DOF cable-driven upper limb exoskeleton," Journal of Mechanical Science and Technology, vol. 32, no. 2, pp. 855-864, 2018.

[16] T. Proietti, V. Crocher, A. Roby-Brami, and N. Jarrasse, "Upper-limb robotic exoskeletons for neurorehabilitation: a review on control strategies," IEEE Reviews in Biomedical Engineering, vol. 9, pp. 4-14, 2016.

[17] F. Zhang, L. Lin, L. Yang, and Y. Fu, "Design of an active and passive control system of hand exoskeleton for rehabilitation," Applied Sciences, vol. 9, no. 11, p. 2291, 2019.

[18] O. Sandoval-Gonzalez, J. Jacinto-Villegas, I. Herrera-Aguilar et al., "Design and development of a hand exoskeleton robot for active and passive rehabilitation," International Journal of Advanced Robotic Systems, vol. 13, no. 2, p. 66, 2016.

[19] W. Yu and J. Rosen, "A novel linear PID controller for an upper limb exoskeleton," in Proceedings of the 2010 49th IEEE
Conference on Decision and Control (CDC), pp. 3548-3553, IEEE, Atlanta, GA, USA, December 2010.

[20] W. Yu and J. Rosen, "Neural PID control of robot manipulators with application to an upper limb exoskeleton," IEEE Transactions on Cybernetics, vol. 43, no. 2, pp. 673-684, 2013.

[21] M. H. Rahman, M. Saad, J.-P. Kenné, and P. S. Archambault, "Control of an exoskeleton robot arm with sliding mode exponential reaching law," International Journal of Control, Automation and Systems, vol. 11, no. 1, pp. 92-104, 2013.

[22] Z. Li, C.-Y. Su, G. Li, and H. Su, "Fuzzy approximation-based adaptive backstepping control of an exoskeleton for human upper limbs," IEEE Transactions on Fuzzy Systems, vol. 23, no. 3, pp. 555-566, 2015.

[23] K. Kiguchi and Y. Hayashi, "An EMG-based control for an upper-limb power-assist exoskeleton robot," IEEE Transactions on Systems, Man, and Cybernetics, Part B (Cybernetics), vol. 42, no. 4, pp. 1064-1071, 2012.

[24] F. Xiao, Y. Wang, Y. Gao, J. Zhu, and J. Zhao, "Continuous estimation of elbow joint angle by multiple features of surface electromyographic using grey features weighted support vector machine," Journal of Medical Imaging and Health Informatics, vol. 7, no. 3, pp. 574-583, 2017.

[25] C. Yang, H. Wu, Z. Li, W. He, N. Wang, and C.-Y. Su, "Mind control of a robotic arm with visual fusion technology," IEEE Transactions on Industrial Informatics, vol. 14, no. 9, pp. 3822-3830, 2017.

[26] C. Yang, Y. Jiang, Z. Li, W. He, and C.-Y. Su, "Neural control of bimanual robots with guaranteed global stability and motion precision," IEEE Transactions on Industrial Informatics, vol. 13, no. 3, pp. 1162-1171, 2017.

[27] W. He, Y. Chen, and Z. Yin, "Adaptive neural network control of an uncertain robot with full-state constraints," IEEE Transactions on Cybernetics, vol. 46, no. 3, pp. 620-629, 2016.

[28] C. Cortés, A. de los Reyes-Guzmán, D. Scorza et al., "Inverse kinematics for upper limb compound movement estimation in exoskeleton-assisted rehabilitation," BioMed Research International, vol. 2016, Article ID 2581924, 14 pages, 2016.

[29] K. Anam and A. A. Al-Jumaily, "Active exoskeleton control systems: state of the art," Procedia Engineering, vol. 41, pp. 988-994, 2012.

[30] W. He, Y. Dong, and C. Sun, "Adaptive neural impedance control of a robotic manipulator with input saturation," IEEE Transactions on Systems, Man, and Cybernetics: Systems, vol. 46, no. 3, pp. 334-344, 2016.

[31] H.-B. Kang and J.-H. Wang, "Adaptive robust control of 5 DOF Upper-limb exoskeleton robot," International Journal of Control, Automation and Systems, vol. 13, no. 3, pp. 733-741, 2015.

[32] F. Antonio, S. Fabio, B. Massimo, B. Rossi, and M. C. Carboncini, "A force-feedback exoskeleton for upper-limb rehabilitation in virtual reality," Applied Bionics and Biomechanics, vol. 6, no. 2, pp. 115-126, 2009.

[33] Y. Chen, G. Li, Y. Zhu, J. Zhao, and H. Cai, "Design of a 6DOF upper limb rehabilitation exoskeleton with parallel actuated joints," Bio-Medical Materials and Engineering, vol. 24, no. 6, pp. 2527-2535, 2014.

[34] G. Li, Q. Fang, T. Xu, J. Zhao, H. Cai, and Y. Zhu, "Inverse kinematic analysis and trajectory planning of a modular upper limb rehabilitation exoskeleton," Technology and Health Care, vol. 27, pp. 123-132, 2019.

[35] J. J. Craig, Introduction to Robotics: Mechanics and Control, Pearson Education India, Bangalore, Karnataka, India, 2009.

[36] L. L. Tien, A. Albu-schäffer, and G. Hirzinger, "MIMO state feedback controller for a flexible joint robot with strong joint 
coupling," in Proceedings of the IEEE International Conference on Robotics \& Automation, IEEE, Roma, Italy, April 2007.

[37] T. Xu, J. Fan, Q. Fang, S. Wang, Y. Zhu, and J. Zhao, "A novel virtual sensor for estimating robot joint total friction based on total momentum," Applied Sciences, vol. 9, no. 16, p. 3344, 2019.

[38] B. Siciliano, L. Sciavicco, L. Villani, and G. Oriolo, Robotics: Modelling, Planning and Control, Springer Science \& Business Media, New York, USA, 2010.

[39] H. S. Lo: Exoskeleton Robot for Upper Limb Rehabilitation: Design Analysis and Control, ResearchSpace@ Auckland, Auckland, New Zealand, 2014. 


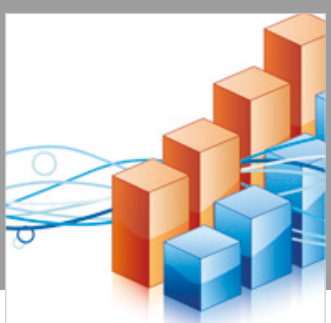

Advances in

Operations Research

\section{-n-m}
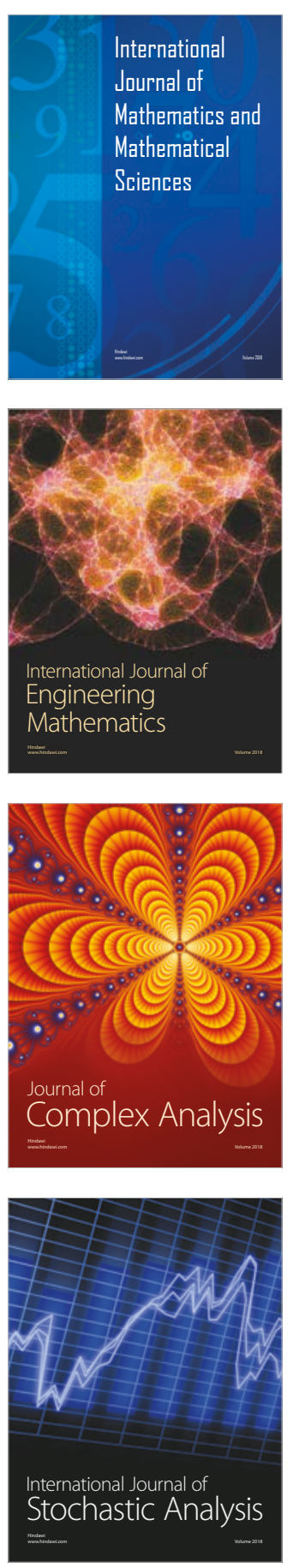
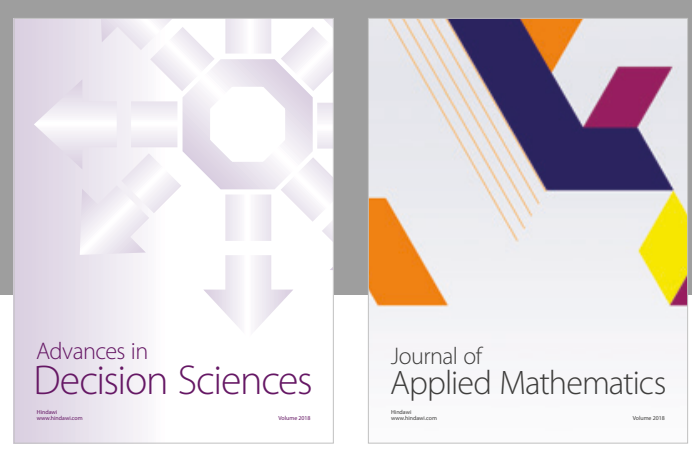

Journal of

Applied Mathematics
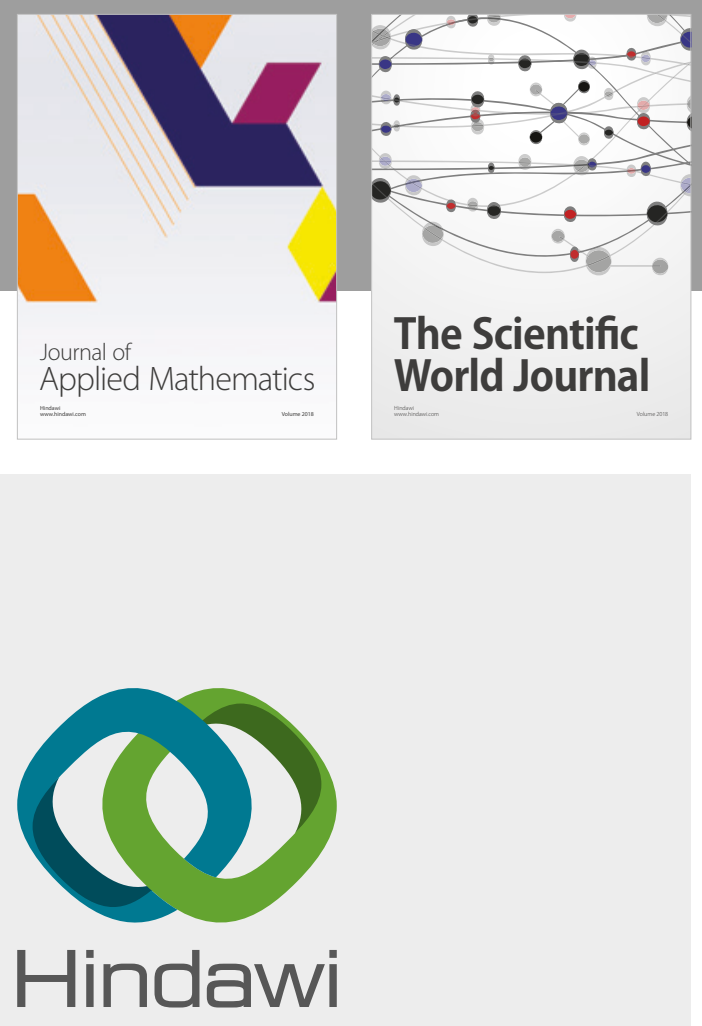

Submit your manuscripts at

www.hindawi.com

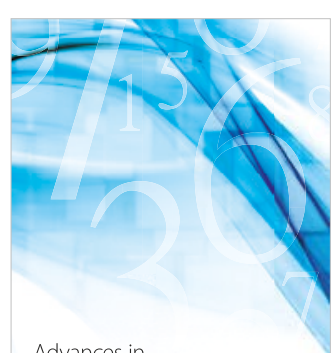

Advances in
Numerical Analysis
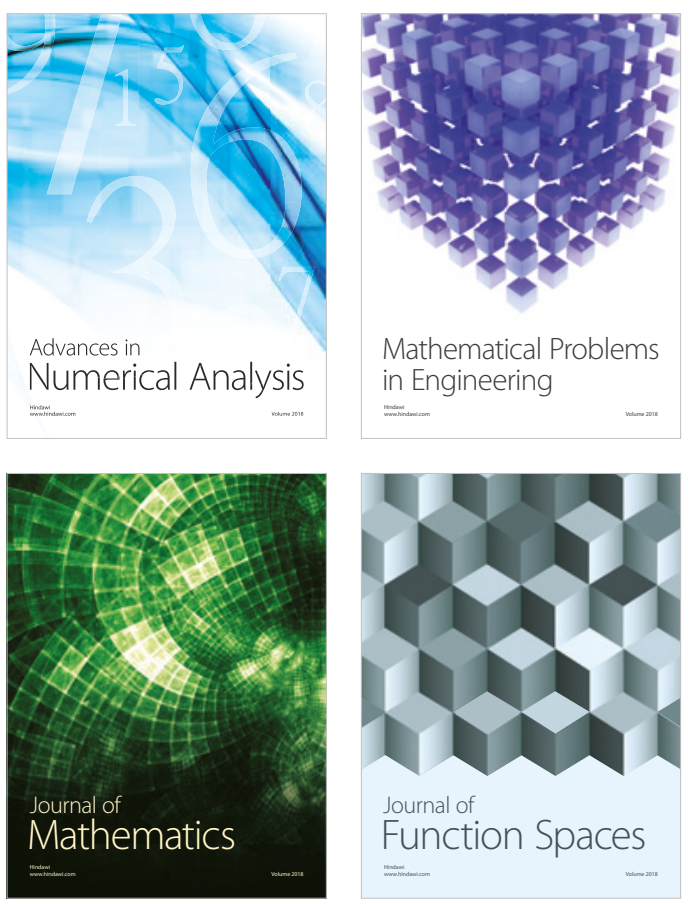

Mathematical Problems in Engineering

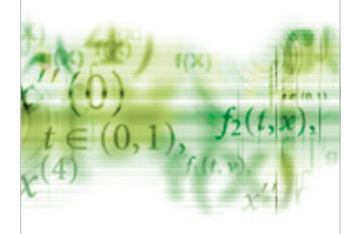

International Journal of

Differential Equations

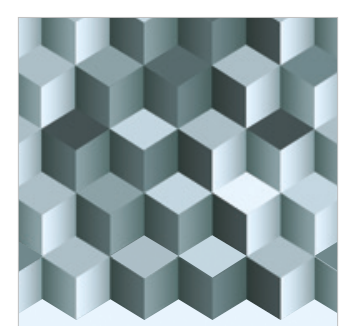

Journal of

Function Spaces

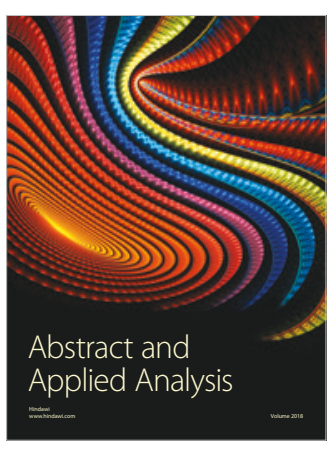

The Scientific

World Journal

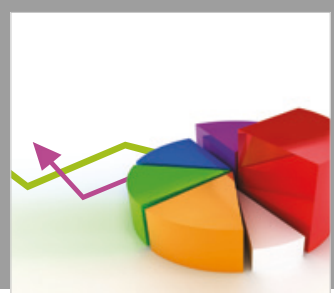

Journal of

Probability and Statistics
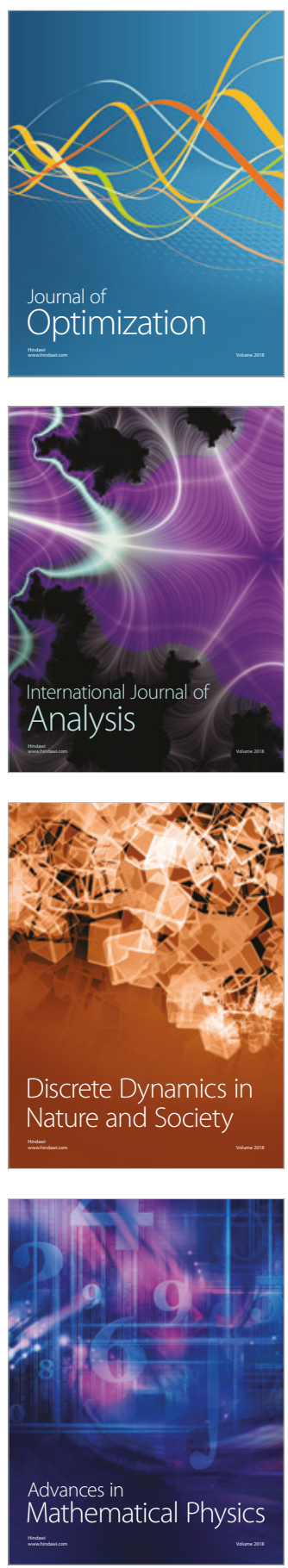